\title{
LINEARIZATION METHOD FOR SOLVING NONLINEAR INTEGRAL EQUATIONS
}

\author{
P. DARANIA, A. EBADIAN, AND A. V. OSKOI \\ Received 16 May 2005; Revised 12 September 2005; Accepted 23 October 2005
}

The objective of this paper is to assess both the applicability and the accuracy of linearization method in several problems of general nonlinear integral equations. This method provides piecewise linear integral equations which can be easily integrated. It is shown that the accuracy of linearization method can be substantially improved by employing variable steps which adjust themselves to the solution. This approach can reveal that, under this method, the nonlinear integral equations can be transformed into the linear integral equations which may be integrated using classical methods. Numerical examples are used to illustrate the preciseness and effectiveness of the proposed method.

Copyright (C) 2006 P. Darania et al. This is an open access article distributed under the Creative Commons Attribution License, which permits unrestricted use, distribution, and reproduction in any medium, provided the original work is properly cited.

\section{Introduction}

Modeling and analysis of physical phenomena in applied sciences often generates nonlinear mathematical problems. Nonlinearity may be an inner feature of the model, that is, evolution equations with nonlinear terms, or of the problem, that is, nonlinear boundary conditions. The interplay between applied sciences and mathematics then leads to the development of initial and/or boundary value problems for nonlinear partial differential or integral or integrodifferential equations modeling real physical systems. The theory and application of integral equations is an important subject within applied mathematics. Integral equations are used as mathematical models for many and varied physical situations, and also occur as reformulations of other mathematical problems. Since many physical problems are modeled by integral equations, the numerical solutions of such integral equations have been highly studied by many authors. In recent years, numerous works have been focusing on the development of more advanced and efficient methods for integral equations such as implicitly linear collocation methods [1], product integration method [3], and Hermite-type collocation method [2] and semianalytical-numerical techniques such as Adomian's decomposition method [4].

Hindawi Publishing Corporation

Mathematical Problems in Engineering

Volume 2006, Article ID 73714, Pages 1-10

DOI 10.1155/MPE/2006/73714 


\section{On the numerical solutions of integral equations}

The linearization method presented in this paper for integral equations is based on the piecewise linearization of the nonlinear integral equations and the analytical solution of the resulting linear integral equation. Thus, linearization methods provide closed-form solutions in a piecewise fashion and are not iterative; therefore, they do not need a judicious guess to the solution. Furthermore, since these methods are based on the linearization of the nonlinear integral equations, they also provide a means to adapt the step size to the solution. If one is only interested in the values of the dependent variables at discrete values of the independent variable, linearization methods provide explicit nonlinear mappings.

\section{Model problems and method}

We will consider the general nonlinear Volterra integral equation of the form

$$
y(x)=f(x)+\lambda \int_{a}^{x} K(x, t, y(t)) d t
$$

where $y(x)$ is an unknown function, $a$ is a real constant, and the functions $f(x)$ and $K(x, t, y(t))$ are analytical on $\mathbf{R}$ and $\mathbf{R}^{3}$, respectively.

Note that $K(x, t, y(t))$ is a nonlinear function which may be singular at $x=x_{0}$.

Now, we are interested to find the numerical solution to $(2.1)$ in domain $T=[a,+\infty)$. Consider the interval $[a, b) \subseteq T$, and divide it into a series of subintervals $\left[x_{n}, x_{n+1}\right)$ such that $x_{0}=a$. In each subinterval $K(x, t, y)$ may be linearized as follows.

(1) If $K(x, t, y)$ is regular, $K$ may be approximated by the first three terms of its Taylor series expansion around $\left(x_{n}, t_{n}, y_{n}\right)$ in the following form:

$$
\begin{aligned}
K(x, t, y)= & K\left(x_{n}, t_{n}, y_{n}\right)+\left(x-x_{n}\right) \frac{\partial K\left(x_{n}, t_{n}, y_{n}\right)}{\partial x} \\
& +\left(t-t_{n}\right) \frac{\partial K\left(x_{n}, t_{n}, y_{n}\right)}{\partial t}+\left(y-y_{n}\right) \frac{\partial K\left(x_{n}, t_{n}, y_{n}\right)}{\partial y} .
\end{aligned}
$$

By substituting (2.2) in (2.1), we obtain

$$
y(x)=f(x)+\lambda \int_{a}^{x}\left[K_{n}+\left(x-x_{n}\right) J_{n}+\left(t-t_{n}\right) Q_{n}+\left(y-y_{n}\right) Z_{n}\right] d t, \quad x_{n} \leq x<x_{n+1},
$$

where

$$
\begin{gathered}
y\left(x_{n}\right)=y_{n}, \\
K_{n}=K\left(x_{n}, t_{n}, y_{n}\right), \quad J_{n}=\frac{\partial K\left(x_{n}, t_{n}, y_{n}\right)}{\partial x}, \\
Q_{n}=\frac{\partial K\left(x_{n}, t_{n}, y_{n}\right)}{\partial t}, \quad Z_{n}=\frac{\partial K\left(x_{n}, t_{n}, y_{n}\right)}{\partial y} .
\end{gathered}
$$


Since in the integral part of (2.3) $t$ is an independent variable, $y$ is a dependent variable, and $x$ is a parameter, therefore by integrating it respect to $t$, we have

$$
y(x)=f(x)+\lambda Z_{n} \int_{a}^{x} y(t) d t+\lambda\left[K_{n}+\left(x-x_{n}\right) J_{n}-y_{n} Z_{n}\right] \int_{a}^{x} d t+\lambda Q_{n} \int_{a}^{x}\left(t-t_{n}\right) d t .
$$

From this equation and after some computations, we obtain

$$
\begin{aligned}
y(x)= & f(x)+\lambda\left[K_{n}+\left(x-x_{n}\right) J_{n}-y_{n} Z_{n}\right](x-a) \\
& +\frac{\lambda}{2}\left[\left(x-t_{n}\right)^{2}-\left(a-t_{n}\right)^{2}\right] Q_{n}+\lambda Z_{n} \int_{a}^{x} y(t) d t, \quad x_{n} \leq x<x_{n+1} .
\end{aligned}
$$

With differentiating (2.7), respect to $x$, we obtain

$$
y^{\prime}(x)-\lambda Z_{n} y(x)=f^{\prime}(x)+\lambda\left[K_{n}+\left(2 x-x_{n}-a\right) J_{n}-y_{n} Z_{n}+Q_{n}\left(x-t_{n}\right)\right] .
$$

It is clear that, this equation is a linear ordinary differential equation, whose analytical solution may be written as

$$
\begin{aligned}
y(x)= & y\left(x_{n}\right)+f(x)+\lambda Z_{n} \exp \left(\lambda Z_{n} x\right) \int_{x_{n}}^{x} f(t) \exp \left(-\lambda Z_{n} t\right) d t \\
- & \frac{1}{Z_{n}}\left\{K_{n}+\left[\left(x-x_{n}\right)+(x-a)+\frac{2}{\lambda Z_{n}}\right] J_{n}+\left(x-t_{n}+\frac{1}{\lambda Z_{n}}\right) Q_{n}\right\} \\
+ & \exp \left(\lambda Z_{n}\left(x-x_{n}\right)\right)\left\{\frac { 1 } { Z _ { n } } \left[K_{n}+\left(x_{n}-a+\frac{2}{\lambda Z_{n}}\right) J_{n}\right.\right. \\
& \left.\left.+\left(x_{n}-t_{n}+\frac{1}{\lambda Z_{n}}\right) Q_{n}\right]-f\left(x_{n}\right)\right\}, \quad x_{n} \leq x<x_{n+1} .
\end{aligned}
$$

Equation (2.9) provides the following nonlinear mapping

$$
\begin{aligned}
& y\left(x_{n+1}\right)= y\left(x_{n}\right)+f\left(x_{n+1}\right)+\lambda Z_{n} \exp \left(\lambda Z_{n} x_{n+1}\right) \int_{x_{n}}^{x_{n+1}} f(t) \exp \left(-\lambda Z_{n} t\right) d t \\
&- \frac{1}{Z_{n}}\left\{K_{n}+\left[\left(x_{n+1}-x_{n}\right)+\left(x_{n+1}-a\right)+\frac{2}{\lambda Z_{n}}\right] J_{n}+\left(x_{n+1}-t_{n}+\frac{1}{\lambda Z_{n}}\right) Q_{n}\right\} \\
&+\exp \left(\lambda Z_{n}\left(x_{n+1}-x_{n}\right)\right)\left\{\frac { 1 } { Z _ { n } } \left[K_{n}+\left(x_{n}-a+\frac{2}{\lambda Z_{n}}\right) J_{n}\right.\right. \\
&\left.\left.+\left(x_{n}-t_{n}+\frac{1}{\lambda Z_{n}}\right) Q_{n}\right]-f\left(x_{n}\right)\right\} .
\end{aligned}
$$

Note that, since the integral equation (2.1) is one-dimensional and $x$ is independent variable, therefore $x$ and $t$ are equivalent. Now, we will use (2.10) and initial condition 
4 On the numerical solutions of integral equations

(2.4) for solving (2.1) by taking $t_{n}=x_{n}$. By this assumption, the numerical solution of (2.1) at the grid points $x_{n+1}$, for $n=0,1,2, \ldots$, can be obtained from

$$
\begin{aligned}
y_{n+1}= & y_{n}+f_{n+1}+\lambda Z_{n} \exp \left(\lambda Z_{n} x_{n+1}\right) \int_{x_{n}}^{x_{n+1}} f(t) \exp \left(-\lambda Z_{n} t\right) d t \\
& -\frac{1}{Z_{n}}\left\{K_{n}+\left[\triangle x_{n}+\left(x_{n+1}-x_{0}\right)+\frac{2}{\lambda Z_{n}}\right] J_{n}+\left(\triangle x_{n}+\frac{1}{\lambda Z_{n}}\right) Q_{n}\right\} \\
& +\exp \left(\lambda Z_{n} \triangle x_{n}\right)\left\{\frac{1}{Z_{n}}\left[K_{n}+\left(x_{n}-x_{0}+\frac{2}{\lambda Z_{n}}\right) J_{n}+\frac{1}{\lambda Z_{n}} Q_{n}\right]-f\left(x_{n}\right)\right\},
\end{aligned}
$$

where $\triangle x_{n}=x_{n+1}-x_{n}$ is the local size step and $y\left(x_{0}\right)=y_{0}$.

On the other hand, if we set $\triangle x_{n}=h$ for $n=0,1,2, \ldots$, namely, $x_{n}=x_{0}+n h$, then (2.11) will be reduced to the following equation:

$$
\begin{aligned}
y_{n+1}= & y_{n}+f_{n+1}+\lambda Z_{n} \exp \left(\lambda Z_{n} x_{n+1}\right) \int_{x_{n}}^{x_{n+1}} f(t) \exp \left(-\lambda Z_{n} t\right) d t \\
& -\frac{1}{Z_{n}}\left\{K_{n}+\left[(n+2) h+\frac{2}{\lambda Z_{n}}\right] J_{n}+\left(h+\frac{1}{\lambda Z_{n}}\right) Q_{n}\right\} \\
& +\exp \left(\lambda Z_{n} h\right)\left\{\frac{1}{Z_{n}}\left[K_{n}+\left(n h+\frac{2}{\lambda Z_{n}}\right) J_{n}+\frac{1}{\lambda Z_{n}} Q_{n}\right]-f\left(x_{n}\right)\right\} .
\end{aligned}
$$

(2) If $K(x, t, y(t))$ is singular at $x_{n}$, the above derivation is not valid, but $K(x, t, y)$ may be approximated by the first three terms of its Taylor series expansion around $\left(x_{n+1}, t_{n+1}\right.$, $y_{n}$ ), so (2.1) may be approximated by

$$
\begin{aligned}
y(x)= & f(x)+\lambda\left[K_{n+1}+\left(x-x_{n+1}\right) J_{n+1}-y_{n} Z_{n+1}\right](x-a) \\
& +\frac{\lambda}{2}\left[\left(x-t_{n+1}\right)^{2}-\left(a-t_{n+1}\right)^{2}\right] Q_{n+1}+\lambda Z_{n+1} \int_{a}^{x} y(t) d t, \quad x_{n}<x \leq x_{n+1},
\end{aligned}
$$

where

$$
\begin{gathered}
K_{n+1}=K\left(x_{n+1}, t_{n+1}, y_{n}\right), \quad J_{n+1}=\frac{\partial K\left(x_{n+1}, t_{n+1}, y_{n}\right)}{\partial x}, \\
Q_{n+1}=\frac{\partial K\left(x_{n+1}, t_{n+1}, y_{n}\right)}{\partial t}, \quad Z_{n+1}=\frac{\partial K\left(x_{n+1}, t_{n+1}, y_{n}\right)}{\partial y}, \\
y\left(x_{n}\right)=y_{n},
\end{gathered}
$$


P. Darania et al. 5

whose analytical solution may be expressed as

$$
\begin{aligned}
& y(x)=y\left(x_{n}\right)+ f(x)+\lambda Z_{n+1} \exp \left(\lambda Z_{n+1} x\right) \int_{x_{n}}^{x} f(t) \exp \left(-\lambda Z_{n+1} t\right) d t \\
&-\frac{1}{Z_{n+1}}\left\{K_{n+1}+\left(2 x-x_{n+1}-a+\frac{2}{\lambda Z_{n+1}}\right) J_{n+1}\right. \\
&\left.+\left(x-t_{n+1}+\frac{1}{\lambda Z_{n+1}}\right) Q_{n+1}\right\}+\exp \left(\lambda Z_{n+1}\left(x-x_{n}\right)\right) \\
& \times\left\{\frac { 1 } { Z _ { n + 1 } } \left[K_{n+1}+\left(2 x_{n}-x_{n+1}-a+\frac{2}{\lambda Z_{n+1}}\right) J_{n+1}\right.\right. \\
&\left.\left.\quad+\left(x_{n}-t_{n+1}+\frac{1}{\lambda Z_{n+1}}\right) Q_{n+1}\right]-f\left(x_{n}\right)\right\}, \quad x_{n}<x \leq x_{n+1} .
\end{aligned}
$$

From (2.15), solution of (2.1) at the grid points $x_{n+1}$ can be obtained from

$$
\begin{aligned}
y_{n+1}= & y_{n}+f_{n+1}+\lambda Z_{n+1} \exp \left(\lambda Z_{n+1} x_{n+1}\right) \int_{x_{n}}^{x_{n+1}} f(t) \exp \left(-\lambda Z_{n+1} t\right) d t \\
- & \frac{1}{Z_{n+1}}\left\{K_{n+1}+\left(x_{n+1}-x_{0}+\frac{2}{\lambda Z_{n+1}}\right) J_{n+1}+\frac{1}{\lambda Z_{n+1}} Q_{n+1}\right\} \\
+ & \exp \left(\lambda Z_{n+1} \triangle x_{n}\right)\left\{\frac { 1 } { Z _ { n + 1 } } \left[K_{n+1}+\left(x_{n}-\Delta x_{n}-x_{0}+\frac{2}{\lambda Z_{n+1}}\right) J_{n+1}\right.\right. \\
& \left.\left.+\left(\frac{1}{\lambda Z_{n+1}}-\Delta x_{n}\right) Q_{n+1}\right]-f\left(x_{n}\right)\right\},
\end{aligned}
$$

where $\triangle x_{n}$, and $t_{n+1}$ are as above.

In the similar manner, if we set $\triangle x_{n}=h$, for $n=0,1,2, \ldots,\left(x_{n}=x_{0}+n h\right)$, then (2.16) will be reduced to the following equation:

$$
\begin{gathered}
y_{n+1}=y_{n}+f_{n+1}+\lambda Z_{n+1} \exp \left(\lambda Z_{n+1} x_{n+1}\right) \int_{x_{n}}^{x_{n+1}} f(t) \exp \left(-\lambda Z_{n+1} t\right) d t \\
-\frac{1}{Z_{n+1}}\left\{K_{n+1}+\left((n+1) h+\frac{2}{\lambda Z_{n+1}}\right) J_{n+1}+\frac{1}{\lambda Z_{n+1}} Q_{n+1}\right\} \\
+\exp \left(\lambda Z_{n+1} h\right)\left\{\frac { 1 } { Z _ { n + 1 } } \left[K_{n+1}+\left((n-1) h+\frac{2}{\lambda Z_{n+1}}\right) J_{n+1}\right.\right. \\
\left.\left.+\left(\frac{1}{\lambda Z_{n+1}}-h\right) Q_{n+1}\right]-f\left(x_{n}\right)\right\} .
\end{gathered}
$$

The nonlinear mappings or difference equations corresponding to (2.12) and (2.17) have been derived by considering that $K$ is a function of three variables $x, t$, and $y$. 
6 On the numerical solutions of integral equations

Moreover, since these equations have been obtained by approximating $K$ by the first three terms of its Taylor series expansion, while higher-order terms have been neglected, the local step size $\triangle x_{n}$ may be determined from the condition that higher-order terms be much smaller than the first-order ones, or from the condition that $y_{n+1}$ does not differ significantly from $y_{n}$.

\section{Error analysis}

In this section, we perform the estimating error for the integral equations. Since the truncated nonlinear mappings or difference equations corresponding to (2.12) and (2.17) are an approximate solution of (2.1), hence, the error function $e(x)$ for (2.1) is defined as follows:

$$
e(x)=|y(x)-Y(x)|
$$

where in the regular point $x_{n}$, we have

$$
\begin{aligned}
Y(x)= & y\left(x_{n}\right)+f(x)+\lambda Z_{n} \exp \left(\lambda Z_{n} x\right) \int_{x_{n}}^{x} f(t) \exp \left(-\lambda Z_{n} t\right) d t \\
- & \frac{1}{Z_{n}}\left\{K_{n}+\left[\left(x-x_{n}\right)+(x-a)+\frac{2}{\lambda Z_{n}}\right] J_{n}+\left(x-t_{n}+\frac{1}{\lambda Z_{n}}\right) Q_{n}\right\} \\
+ & \exp \left(\lambda Z_{n}\left(x-x_{n}\right)\right)\left\{\frac { 1 } { Z _ { n } } \left[K_{n}+\left(x_{n}-a+\frac{2}{\lambda Z_{n}}\right) J_{n}\right.\right. \\
& \left.\left.+\left(x_{n}-t_{n}+\frac{1}{\lambda Z_{n}}\right) Q_{n}\right]-f\left(x_{n}\right)\right\}, \quad x_{n} \leq x<x_{n+1},
\end{aligned}
$$

and in the singular point $x_{n}$, we have

$$
\begin{aligned}
Y(x)=y\left(x_{n}\right)+ & f(x)+\lambda Z_{n+1} \exp \left(\lambda Z_{n+1} x\right) \int_{x_{n}}^{x} f(t) \exp \left(-\lambda Z_{n+1} t\right) d t \\
-\frac{1}{Z_{n+1}}\{ & K_{n+1}+\left(2 x-x_{n+1}-a+\frac{2}{\lambda Z_{n+1}}\right) J_{n+1} \\
& \left.+\left(x-t_{n+1}+\frac{1}{\lambda Z_{n+1}}\right) Q_{n+1}\right\}+\exp \left(\lambda Z_{n+1}\left(x-x_{n}\right)\right) \\
\times\left\{\frac { 1 } { Z _ { n + 1 } } \left[K_{n+1}+\left(2 x_{n}-x_{n+1}-a+\frac{2}{\lambda Z_{n+1}}\right) J_{n+1}\right.\right. & \left.\left.\quad+\left(x_{n}-t_{n+1}+\frac{1}{\lambda Z_{n+1}}\right) Q_{n+1}\right]-f\left(x_{n}\right)\right\}, \quad x_{n}<x \leq x_{n+1} .
\end{aligned}
$$


Now, by substituting the solutions $y\left(x_{r}\right)(r=0,1, \ldots)$ in the error function $e(x)$ we have

$$
e\left(x_{r}\right)=\left|y\left(x_{r}\right)-Y\left(x_{r}\right)\right|
$$

then our aim is $e\left(x_{r}\right) \leq 10^{-k_{r}}$ ( $k_{r}$ is any positive integer). If we prescribe $\max \left(10^{-k r}\right)=$ $10^{-k}$, then we decrease the step size $h$ as far as the following inequality holds at each point $x_{r}$ :

$$
e\left(x_{r}\right) \leq 10^{-k}
$$

In other words, by decreasing $h$ the error function $e\left(x_{r}\right)$ approaches to zero.

\section{Presentation of results}

In order to assess both the applicability and accuracy of the theoretical results of Section 2, we have applied them to a variety of nonlinear integral equations in the following examples.

Example 4.1. The nonlinear Volterra integral equation in $[0,1]$

$$
y(x)=\exp (x)-\frac{1}{2}(\exp (2 x)-1)+\int_{0}^{x} y^{2}(t) d t
$$

has the following analytical solution $y(x)=\exp (x)$, and therefore, provides an example to verify the accuracy of this method.

Equation (4.1) by using (2.7) is reduced to the linear integral equation in the following form:

$$
y(x)=\exp (x)-\frac{1}{2}(\exp (2 x)-1)-y_{n}^{2} x+2 y_{n} \int_{0}^{x} y(t) d t, \quad x_{n} \leq x<x_{n+1},
$$

and from (2.12), numerical solution of (4.2) at the grid points $x_{n+1}, n=0,1,2, \ldots$, can be obtained from

$$
\begin{aligned}
y_{n+1}= & \frac{y_{n}}{2}+\exp \left(x_{n+1}\right)-\frac{1}{2}\left(\exp \left(2 x_{n+1}\right)-1\right) \\
& +\exp \left(2 y_{n} h\right)\left(\frac{y_{n}}{2}-\exp \left(x_{n}\right)+\frac{1}{2}\left(\exp \left(2 x_{n}\right)-1\right)\right) \\
& +2 y_{n} \exp \left(2 y_{n} x_{n+1}\right) \int_{x_{n}}^{x_{n+1}}\left(\exp (t)-\frac{1}{2}(\exp (2 t)-1)\right) \exp \left(-2 y_{n} t\right) d t .
\end{aligned}
$$




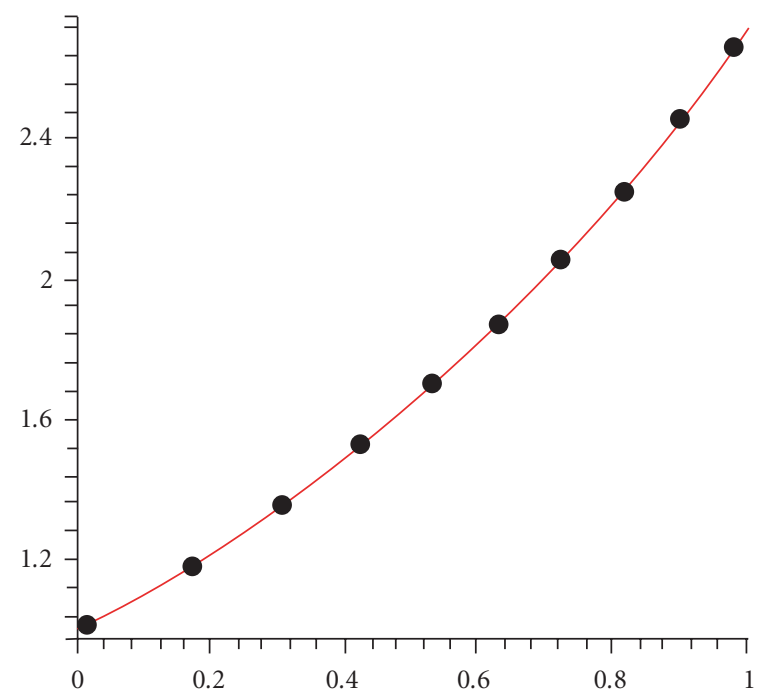

Figure 4.1. The result for $h=0.1$ and 0.01 and analytical solution of (4.1).

The result for $h=0.1$ and 0.01 and analytical solution are presented in Figure 4.1 which indicates that the numerical solutions obtained from (4.3) and step sizes equal to $0.0001,0.001,0.01$, and 0.1 are nearly identical. The exact solution at $x=0.7$ is 2.013752707000 , whereas the numerical solutions corresponding to step sizes equal to $0.0001,0.001,0.01$, and 0.1 are $2.0137523240,2.0137511060,2.0136061360$, and 2.0005244930 , respectively. These results indicate that, if we use the time steps smaller than about 0.01 , then we obtain the numerical solutions of minimum error. In other words, by decreasing $h$ the error function $e\left(x_{r}\right) \leq 10^{-5}$.

Table 4.1 shows the errors-involved presented method with $h=0.0001,0.001,0.01$, and 0.1 along with the exact solution.

Example 4.2. Consider the nonlinear weakly singular Volterra-Hammerstein integral equation with algebraic nonlinearity

$$
y(x)=-\frac{x^{4}}{10}+\frac{5}{6} x^{2}+\frac{3}{8}+\int_{0}^{x} \frac{1}{2 x} y^{2}(t) d t, \quad x \in[0,1]
$$

with exact solution $y(x)=x^{2}+1 / 2$ and singular point $x_{0}=0$.

Equation (4.4) by using (2.15) will be reduced to the linear Volterra integral equation in the following form:

$$
y(x)=-\frac{x^{4}}{10}+\frac{5 x^{2}}{6}+\frac{3}{8}-\frac{y_{n}^{2}}{2 x_{n+1}}\left[1+\frac{x-x_{n+1}}{x_{n+1}}\right] x+\frac{y_{n}}{x_{n+1}} \int_{0}^{x} y(t) d t, \quad x_{n}<x \leq x_{n+1},
$$


Table 4.1. Comparison of numerical solution $y_{i}$ and exact solution $y\left(x_{i}\right)$.

\begin{tabular}{ccccc}
\hline$x$ & $h=0.1$ & $h=0.01$ & $h=0.001$ & $h=0.0001$ \\
\hline 0.0 & $0.000000000 e+00$ & $0.000000000 e+00$ & $0.000000000 e+00$ & $0.000000000 e+0$ \\
0.1 & $3.779750000 e-04$ & $4.065000000 e-6$ & $5.100000000 e-08$ & $2.000000000 e-8$ \\
0.2 & $9.402320000 e-4$ & $1.015300000 e-5$ & $1.160000000 e-7$ & $5.800000000 e-8$ \\
0.3 & $1.783404000 e-3$ & $1.933700000 e-5$ & $2.150000000 e-7$ & $8.500000000 e-8$ \\
0.4 & $3.063988000 e-3$ & $3.337100000 e-5$ & $3.700000000 e-7$ & $1.190000000 e-7$ \\
0.5 & $5.042530000 e-3$ & $5.519100000 e-5$ & $6.140000000 e-7$ & $1.890000000 e-7$ \\
0.6 & $8.166182000 e-3$ & $8.989100000 e-5$ & $9.900000000 e-7$ & $2.580000000 e-7$ \\
0.7 & $1.322821400 e-2$ & $1.465710000 e-4$ & $1.601000000 e-6$ & $3.830000000 e-7$ \\
0.8 & $2.168746500 e-2$ & $2.421720000 e-4$ & $2.612000000 e-6$ & $6.260000000 e-7$ \\
0.9 & $3.633225600 e-2$ & $4.095920000 e-4$ & $4.382000000 e-6$ & $9.340000000 e-7$ \\
1.0 & $6.271307700 e-2$ & $7.156940000 e-4$ & $7.651000000 e-6$ & $1.600000000 e-6$ \\
\hline
\end{tabular}

Table 4.2. Comparison of numerical solution $y_{i}$ and exact solution $y\left(x_{i}\right)$.

\begin{tabular}{cccc}
\hline$x$ & $y_{i}$ & $y\left(x_{i}\right)$ & $\left|y\left(x_{i}\right)-y_{i}\right|$ \\
\hline 0.0 & 1.000000000000 & 1.000000000000 & $0.000000000000 e+00$ \\
0.1 & 0.894271248000 & 0.510000000000 & $3.842712480000 e-01$ \\
0.2 & 0.921230883000 & 0.540000000000 & $3.812308830000 e-01$ \\
0.3 & 0.963056591000 & 0.590000000000 & $3.730565910000 e-01$ \\
0.4 & 1.020125776000 & 0.660000000000 & $3.601257760000 e-01$ \\
0.5 & 1.091795751000 & 0.750000000000 & $3.417957510000 e-01$ \\
0.6 & 1.177052811000 & 0.860000000000 & $3.170528110000 e-01$ \\
0.7 & 1.274605512000 & 0.990000000000 & $2.846055120000 e-01$ \\
0.8 & 1.382907920000 & 1.140000000000 & $2.429079200000 e-01$ \\
0.9 & 1.500167373000 & 1.310000000000 & $1.901673730000 e-01$ \\
1.0 & 1.624347620000 & 1.500000000000 & $1.243476200000 e-01$ \\
\hline
\end{tabular}

from (2.17), we get the numerical solution of (4.4) at the grid points $x_{n+1}$ for $n=0,1,2, \ldots$, in the following form:

$$
\begin{aligned}
y_{n+1}= & \frac{y_{n}}{2}\left(1+\frac{(n+1) h}{x_{n+1}}\right)-\frac{x_{n+1}^{4}}{10}+\frac{5}{6} x_{n+1}^{2}+\frac{11}{8} \\
& +\frac{y_{n}}{x_{n+1}} \exp \left(y_{n}\right) \int_{x_{n}}^{x_{n+1}}\left(\left(-\frac{t^{4}}{10}+\frac{5}{6} t^{2}+\frac{3}{8}\right) \exp \left(-\frac{y_{n}}{x_{n+1}} t\right)\right) d t \\
& +\exp \left(\frac{y_{n}}{x_{n+1}} h\right)\left(\frac{y_{n}}{2}\left(1-\frac{(n-1) h}{x_{n+1}}\right)+\frac{x_{n}^{4}}{10}-\frac{5}{6} x_{n}^{2}-\frac{11}{8}\right) .
\end{aligned}
$$

The absolute errors found by presented method are compared with the exact solution in Table 4.2. 


\section{Conclusion}

By introducing the Linearization method, the nonlinear Volterra integral equations in applied sciences and physics can be transformed into the linear Volterra integral equations which may be integrated using classical methods. Both the applicability and the accuracy of linearization method for the solution of nonlinear integral equations have been examined by means of several problems. It has been shown that, for regular problems, linearization method is robust and accurate techniques whose accuracy is not a strong function of either the linearization point or the linearization with respect to the independent variable.

\section{Acknowledgments}

The authors would like to express their gratitude to Urmia University, Iran, as this research was in part supported by a Grant from Urmia University (no. 003/ayn/84). The authors truly appreciate the comments made by referees.

\section{References}

[1] H. Brunner, Implicitly linear collocation methods for nonlinear Volterra equations, Applied Numerical Mathematics 9 (1992), no. 3-5, 235-247.

[2] T. Diogo, S. McKee, and T. Tang, A Hermite-type collocation method for the solution of an integral equation with a certain weakly singular kernel, IMA Journal of Numerical Analysis 11 (1991), no. 4, 595-605.

[3] T. Tang, S. McKee, and T. Diogo, Product integration methods for an integral equation with logarithmic singular kernel, Applied Numerical Mathematics 9 (1992), no. 3-5, 259-266.

[4] A.-M. Wazwaz and S. M. El-Sayed, A new modification of the Adomian decomposition method for linear and nonlinear operators, Applied Mathematics and Computation 122 (2001), no. 3, 393-405.

P. Darania: Department of Mathematics, Faculty of Science, Urmia University,

P.O. Box 165, Urmia, Iran.

E-mail address: p.darania@mail.urmia.ac.ir

A. Ebadian: Department of Mathematics, Faculty of Science, Urmia University,

P.O. Box 165, Urmia, Iran.

E-mail address: a.ebadian@mail.urmia.ac.ir

A. V. Oskoi: Department of Mathematics, Faculty of Science, Urmia University,

P.O. Box 165, Urmia, Iran.

E-mail address: a.v.oskoi@mail.urmia.ac.ir 


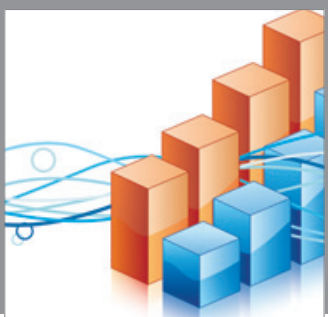

Advances in

Operations Research

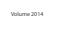

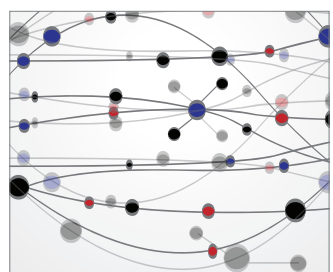

\section{The Scientific} World Journal
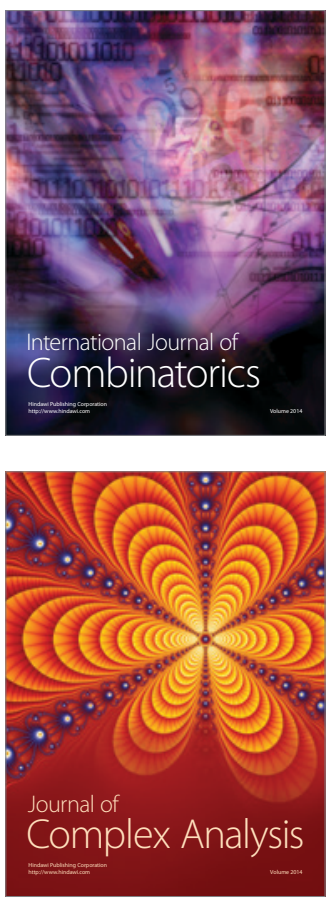

International Journal of

Mathematics and

Mathematical

Sciences
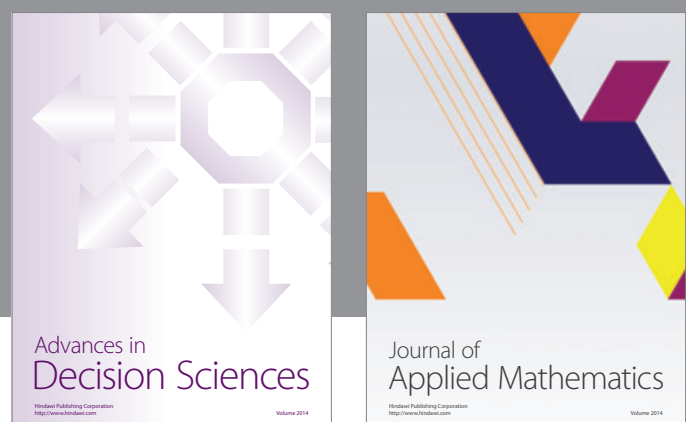

Journal of

Applied Mathematics
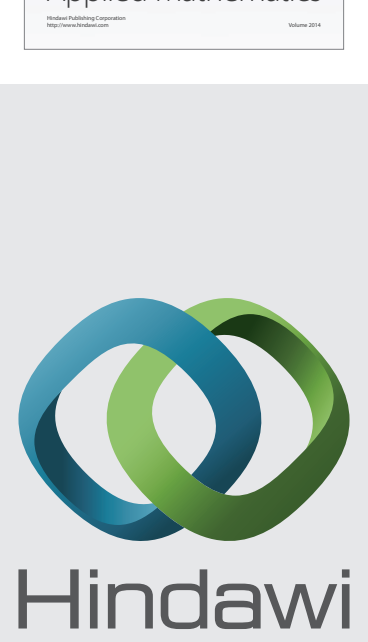

Submit your manuscripts at http://www.hindawi.com
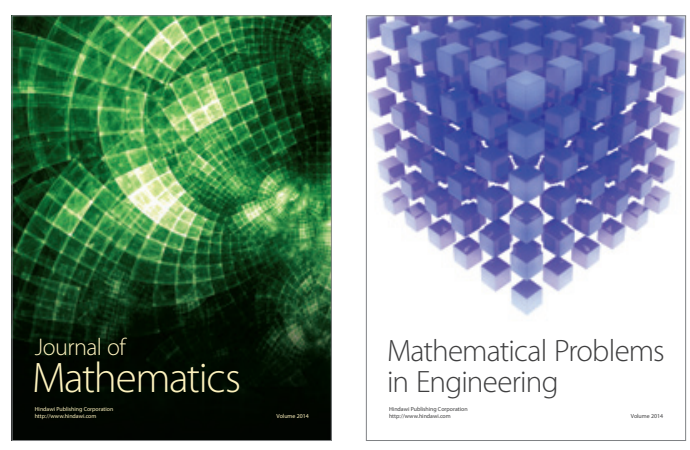

Mathematical Problems in Engineering
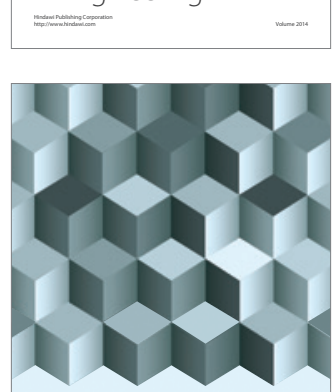

Journal of

Function Spaces
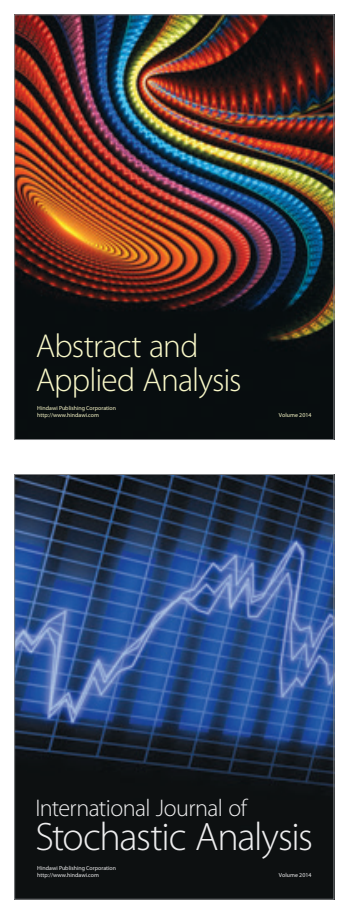

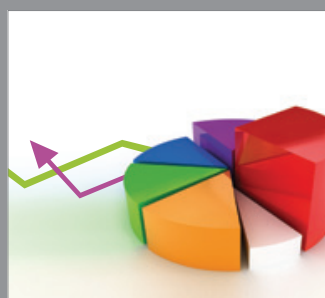

ournal of

Probability and Statistics

Promensencen
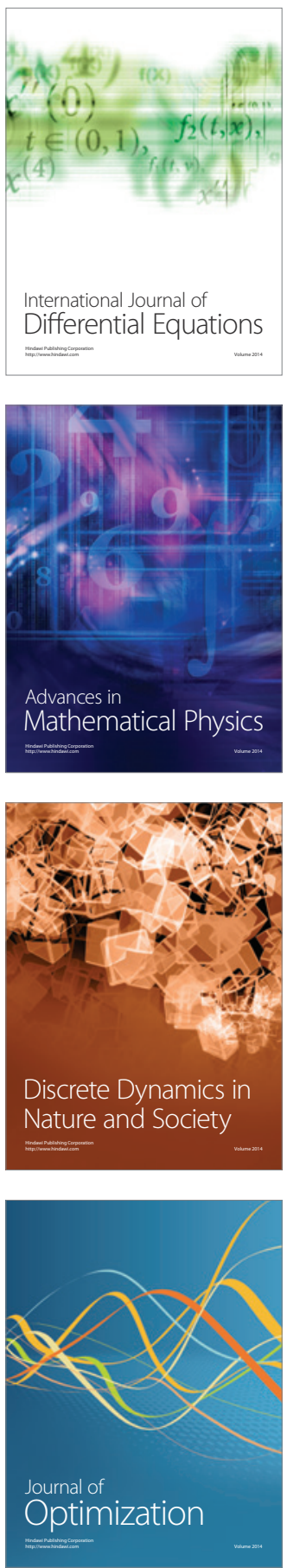